\section{Engagement of brain areas implicated in processing inner speech in people with auditory hallucinations}

\author{
SUKHWINDER S. SHERGILL, MICHAEL J. BR AMMER, RIMMEI FUKUDA, \\ STEVEN C. R. WILLIAMS, ROBIN M. MURRAY and PHILIP K. McGUIRE
}

Auditory hallucinations occur frequently in people with psychiatric disorders, being most common in schizophrenia, and are often described as 'voices'. Such hallucinations are usually treated with antipsychotic medication, but in $25-30 \%$ of patients they are refractory to traditional antipsychotic drugs. Their pathophysiological basis remains unknown, although functional brain imaging studies have demonstrated the involvement of frontal and temporal cortical regions implicated in the generation and perception of verbal material (Shergill et al, 2000a,b).

\section{Self-monitoring}

Self-monitoring is fundamental to normal cognitive function, in planning, controlling and anticipating the consequences of complex motor acts. A feed-forward model has been proposed to explain this mechanism (Wolpert et al, 1995); in this model the motor outflow of a motor act (plan) generates an efferent copy that is transmitted to (sensory) brain regions relevant to this act and serves to anticipate its sensory effects. The anticipated sensory consequences are then subtracted from the actual sensory feedback, which has the consequence of cancelling out the sensory effects of the motor act, thereby leaving sensory information about changes in the outside world. An everyday example is that during self-generated eye movements the world is experienced as stable, despite the succession of images passing the retina. However, when the eye is moved passively by tapping the eyeball, and therefore there is no cancellation of the sensory effects of the motor act, it is the visual world that seems to move. This simple cancellation theory has been superseded by the concept of feed-forward control, whereby the motor commands themselves are monitored, prior to any actual movement (Deeke et al, 1969; Erdler et al, 2000). Further examples of differences in brain activation patterns between identical externally generated and self-generated actions have been reported in the auditory and tactile modalities. The auditory cortices are activated in response to vowel changes in heard speech, but not when the same vowel changes are selfuttered (Curio et al, 2000). This suggests that motor-to-sensory priming of the auditory cortex dampens the response to selfproduced 'expected' sounds and occurs on a millisecond timescale.

\section{Self-monitoring of speech}

Electrophysiological recordings in both non-human (Müller-Preuss \& Ploog, 1981) and human (Creutzfeldt et al, 1989) primates indicate that neuronal activity in the temporal cortex is powerfully modulated by vocalisation. This modulation can precede articulation by hundreds of milliseconds, suggesting that it is related to the intention to speak (rather than articulation per se) and mediated by the direct anatomical connections linking areas that generate and perceive speech, within the left inferior frontal and bilateral temporal cortices respectively. Contemporary cognitive models propose that auditory verbal hallucinations are derived from inner speech that the person has misidentified as 'alien' through defective self-monitoring (Frith \& Done, 1988). This suggests that the functional neuroanatomy of monitoring inner speech might be abnormal in people who are prone to auditory verbal hallucinations.

Functional neuroimaging studies of verbal generation in normal individuals indicate that this process is associated with activation in the left prefrontal cortex and deactivation in the temporal cortex bilaterally (Frith $e t$ al, 1995). These findings suggest that output from regions involved in verbal generation might modulate activation in areas involved in speech perception. Initial studies of verbal fluency in schizophrenia indicated that this temporal deactivation was absent (Frith et al, 1995), leading to the proposal that schizophrenia involves a functional frontotemporal disconnectivity. However, more recent studies of verbal fluency in schizophrenia have failed to replicate this finding (Spence et al, 2000).

Auditory verbal imagery - imagining another person's speech - implicitly involves both the generation and monitoring of inner speech, and in healthy volunteers is associated with activation in the 
left inferior frontal cortex, and the temporal, parahippocampal and cerebellar cortex (Shergill et al, 2001). A positron emission tomography (PET) study of auditory verbal imagery in participants with schizophrenia who were prone to auditory hallucinations revealed normal activation of the left inferior frontal gyrus, but differential activation of the left temporal cortex, compared with both people with schizophrenia but no history of hallucinations and healthy volunteers (McGuire et al, 1996). A more recent functional magnetic resonance imaging (fMRI) study using a similar paradigm in another group of hallucination-prone participants again demonstrated normal activation of the left inferior frontal gyrus and attenuated activation of the right temporal cortex (Shergill et al, 2000b). In addition, there was relatively attenuated activation in the parahippocampal and posterior cerebellar cortex bilaterally. However, while imagining speech engages verbal self-monitoring, activation associated with this process could also be related to the phonological and semantic demands of reproducing a representation of another person's voice (McGuire et al, 1996; Shergill et al, $2000 b$ ). In this study we examined a task that engaged the process of inner speech but did not involve these potentially confounding components.

We used fMRI to study people while they were covertly generating the same word ('rest') at different rates. Increasing the rate of covert articulation was designed to increase the demands on the generation and monitoring of inner speech. We compared a group of people with schizophrenia, who were in remission but had a history of prominent auditory hallucinations, with a matched group of healthy volunteers. On the basis of our previous studies of auditory verbal imagery (McGuire et al, 1996; Shergill et al, $2000 b$ ) we predicted that:

(a) increasing the rate of covert articulation would be associated with activation in the left inferior frontal gyrus, the temporal and parahippocampal cortex, and the cerebellum;

(b) there would be no difference between the two study groups in left inferior frontal activation;

(c) participants with schizophrenia would differ from the comparison group in showing attenuated activation in the temporal, parahippocampal and cerebellar cortex.

\section{METHOD}

\section{Participants}

Eight men with schizophrenia, righthanded according to Annett's scale (Annett, 1970), participated in the study; they were recruited from wards and clinics at the Maudsley Hospital, London. Diagnosis was based on DSM-IV criteria (American Psychiatric Association, 1994), using a detailed clinical interview (by S.S.S.) and review of the hospital case notes. Patients were excluded if they had a history of head injury, neurological symptoms, speech or hearing difficulties, fulfilled DSM-IV criteria for misuse of or dependence on any illicit drugs or alcohol during their lifetime, or had any contraindications to MRI scanning (including metal implants or claustrophobia). The participants' mean age was 31 years (s.d. $=9$, range $20-45$ ) and their mean IQ was 110 (s.d. $=8$, range 99-124), measured using the National Adult Reading Test (Nelson, 1991). Those selected had experienced prominent and frequent auditory verbal hallucinations during previous exacerbations of their illness, but were in clinical remission at the time of the study. Control participants were matched for gender, handedness, age (mean 29 years; s.d. $=5$, range $23-37$ ) and IQ (mean 115; s.d. $=5$, range 110-125); those included did not suffer from any medical or psychiatric disorder, were not receiving medication, and had no family history of psychiatric disorder.

The patients' clinical profile and history at interview were confirmed by liaison with the responsible clinical team and assessment of medical records. All except one person (who was not receiving treatment) had been receiving antipsychotic medication (five were treated with atypical and two with typical antipsychotic drugs) at stable dosages for 4 months or more prior to scanning. Before inclusion, potential participants were assessed on their ability to repeat a word overtly at the two rates (once every $1 \mathrm{~s}$ or $4 \mathrm{~s}$ ) to be used during scanning. They proceeded to scanning when they consistently achieved a 1:4 ratio in the number of repetitions timed over a minute. After a complete description of the study to the participants, their written informed consent to participation was obtained. The study was approved by the Maudsley Hospital ethics committee.

\section{Tasks performed during imaging}

Fast v. slow covert articulation (categorical comparison)

Participants covertly generated the word 'rest' repeatedly at two self-paced rates (once every $1 \mathrm{~s}$ or $4 \mathrm{~s}$; i.e. 60 words/min or 15 words/min), without speaking. During scanning, the two conditions alternated in an $\mathrm{ABAB}$ design, with each condition lasting $30 \mathrm{~s}$, and five cycles of each condition in a $300 \mathrm{~s}$ run. The order of conditions was counterbalanced across the group. The desired rate during each condition was indicated by a number visible throughout in the centre of a computer screen (' 1 ' for one word every second and ' 4 ' for one word every $4 \mathrm{~s}$ ). The ability of participants to generate output at the required rates was demonstrated by asking them to tap their finger at the two different rates both before and immediately after scanning. In order to reduce potentially confounding effects of poor performance on activation, only data from participants who achieved a consistent timing ratio (on finger-tapping) of 1:4 between the fast and slow rate, immediately before and after scanning, were analysed.

\section{Image acquisition}

Gradient-echo echoplanar magnetic resonance images were acquired using a $1.5 \mathrm{~T}$ imaging system fitted with Advanced NMR hardware and software (ANMR, Woburn, MA, USA) at the Maudsley Hospital, London. A quadrature birdcage head coil was used for radiofrequency transmission and reception. In each of 14 non-contiguous planes parallel to the intercommissural (anterior-posterior) plane, $100 \mathrm{~T}_{2}^{*}$-weighted images depicting blood oxygen level dependent (BOLD) contrast (Ogawa et al, 1990) were acquired with echo time $40 \mathrm{~ms}$, repetition time $3000 \mathrm{~ms}$, in-plane resolution $3.1 \mathrm{~mm}$, slice thickness $7 \mathrm{~mm}$, slice skip $0.7 \mathrm{~mm}$. At the same session a 43-slice, high-resolution inversion recovery echoplanar image of the whole brain was acquired in the intercommissural plane (repetition time $16000 \mathrm{~ms}$, in-plane resolution $1.5 \mathrm{~mm}$, slice thickness $3 \mathrm{~mm}$ ).

\section{Image analysis}

Image analysis was performed using Statistical Parametric Mapping version SPM99 (Wellcome Department of 
Cognitive Neurology, http://www.fil.ion. ucl.ac.uk/spm/). All data-sets were automatically realigned to the first image to correct for head movement, normalised using sinc interpolation and transformed into Talairach space. The transformed data-set for each participant was smoothed with a Gaussian filter (full width half maximum, $8 \mathrm{~mm}$ ) to compensate for normal variations in anatomy. The time series were high-pass (126 s) filtered to remove low-frequency artefacts.

\section{Fast v. slow covert articulation}

Statistical analysis was performed separately for each individual, and the stereotaxically normalised fMRI time series data from all the participants were pooled for group analysis. Analysis of the task used a categorical design revealing activation during the fast relative to the slow rate of generation, and vice versa. Cluster-level statistics corrected for multiple comparisons were thresholded at $P<0.05$.

\section{Functional connectivity of the left inferior frontal gyrus}

An analysis of the functional connectivity of the left inferior frontal cortex was also performed. The time series data from the voxel in the left inferior frontal gyrus showing the maximally significant response, in the fast $v$. slow covert articulation, was selected as the covariate of interest in each individual. The left inferior frontal gyrus was chosen because it is the main region implicated in the generation of inner speech (McGuire et al, 1996; Shergill et al, 2000b, 2001). We thus sought to identify brain regions whose activity was temporally correlated with activity in the inferior frontal gyrus, and to compare these between groups. The statistical parametric maps (SPMs) from this exploratory analysis were thresholded at $P<0.05$, with voxellevel statistics corrected for multiple comparisons.

\section{Between-group analyses}

Between-group comparisons assessed the significance of differences in the magnitude of each of the above analyses comparing the patient and control groups, using a random effects model. The random effects analysis takes contrasts from a first-level analysis to a second-level SPM analysis, the condition-specific effects (from all participants) are summarised with a contrast and a two-sample $t$ test is applied to the ensuing individual-specific contrasts at the second level. This analysis treats variations in activation from person to person as a random effect. The SPMs were thresholded at $P<0.05$ with cluster-level statistics corrected for multiple comparisons; we also reported cluster-level statistics uncorrected for multiple comparisons in regions included in our a priori hypotheses.

\section{RESULTS}

\section{Behavioural data}

All participants reported that they were able to perform the tasks during scanning and showed a consistent 1:4 timing ratio (on finger-tapping) both before and after scanning. There was no difference in performance between the patient and control groups, and data from all participants were included in the analysis.

\section{Imaging data: control group}

Fast v. slow covert articulation

The data for the control group have been described in detail elsewhere (Shergill et al, 2002). In brief, relative to covert generation at 15 words/min, covert generation at 60 words/min was associated with activation in foci in the dorsolateral and orbital portions of the left inferior frontal gyrus, and in the anterior part of the left superior temporal gyrus. There was also a large area of activation centred on the right precentral gyrus, which included foci in the adjacent postcentral and superior temporal gyri, and a separate bilateral activation in the frontal pole. Activation was also present in the right parahippocampal gyrus and bilateral cerebellar cortices, significant only at the uncorrected level. The slower rate of generation was associated with activation in the supplementary motor area, the left precentral gyrus and the right inferior parietal lobule.

\section{Functional connectivity of the left inferior frontal gyrus}

Activity in the left inferior frontal gyrus was positively correlated with activity in the left middle and superior temporal gyri, inferior parietal lobule, and claustrum. Right-sided correlations were evident in the homologous portion of the inferior frontal gyrus, the precentral and postcentral gyri, and the middle/superior temporal and anterior cingulate gyri.
Activity in the left inferior frontal gyrus was negatively correlated with activity in the left cerebellar cortex and the right thalamus.

\section{Imaging data: patient group}

Fast v. slow covert articulation

As in the control group, the faster rate was associated with activation in the dorsolateral and orbital portion of the left inferior frontal gyrus, and in the anterior part of the left superior temporal gyrus. Additional activation was evident in bilateral thalami, the right middle frontal gyrus and the supplementary motor area. The slower rate of generation was associated with activation in the parahippocampal gyri bilaterally and in the left posterolateral cerebellum and left inferior occipital gyrus (Table 1).

\section{Functional connectivity of the left inferior frontal gyrus}

Activity in the left inferior frontal gyrus was positively correlated with activity in the left superior temporal gyrus and the right dorsolateral prefrontal, middle temporal and posterolateral cerebellar cortices. Activity in the left inferior frontal gyrus was negatively correlated with activity in the retrosplenial cingulate and right lingual gyri (Table 2).

\section{Between-group differences in activation}

\section{Fast v. slow covert articulation}

Compared with the control group, the participants with schizophrenia showed reduced activation in a large right-sided region with distinct foci in the superior temporal and postcentral gyri, and the inferior parietal lobule. Attenuated activation was also evident in the right parahippocampal gyrus and in the posterolateral cerebellar cortex (Table 3; Fig. 1). Relatively increased activation occurred only in the left lenticular nucleus.

\section{Functional connectivity of the left inferior frontal gyrus}

Relative to the control group; in the patient group there was a reduced correlation with left inferior frontal activity in the right middle and superior temporal gyri, the right insula, a region encompassing the right parahippocampal, inferior temporal 
Table I Regions demonstrating significant activation during faster and slower rates (categorical analysis) in patients

\begin{tabular}{|c|c|c|c|c|c|c|}
\hline Region & $x$ & $Y$ & $Z$ & $\begin{array}{l}\text { Cluster size } \\
\text { (voxels) }\end{array}$ & $Z$ score & $\begin{array}{l}\text { P corrected } \\
\text { (cluster) }\end{array}$ \\
\hline \multicolumn{7}{|l|}{ Greater activation at faster rate } \\
\hline \multicolumn{7}{|l|}{ Left inferior frontal gyrus } \\
\hline (BA 47) & -52 & 34 & -6 & 267 & 4.6 & 0.012 \\
\hline (BA 45/46) & -42 & 32 & 12 & & 4.2 & \\
\hline Right supplementary motor area & 20 & -30 & 66 & 521 & 4.7 & $0.000 \mathrm{I}$ \\
\hline Left middle temporal gyrus (BA 2I) & -52 & -12 & -8 & 931 & 4.8 & 0.0001 \\
\hline Left superior temporal gyrus (BA 42) & -48 & -10 & 8 & & 4.6 & \\
\hline Left thalamus & -4 & -2 & 6 & 209 & 4.9 & 0.03 \\
\hline Right thalamus & 6 & -2 & 6 & & 4.7 & \\
\hline Right middle frontal gyrus (BA 8/9) & 58 & 22 & 34 & 735 & 5.6 & 0.0001 \\
\hline Left lentricular nucleus & -28 & -12 & 6 & 100 & 3.8 & $0.04^{\prime}$ \\
\hline \multicolumn{7}{|l|}{ Greater activation at slower rate } \\
\hline Right parahippocampal gyrus (BA 36) & 30 & -20 & -22 & 210 & 4.8 & 0.03 \\
\hline Posterior cingulate gyrus (BA 30 ) & 0 & -40 & 12 & 1352 & 6.8 & 0.0001 \\
\hline $\begin{array}{l}\text { Right parahippocampal gyrus } \\
\qquad \text { (BA 27/30) }\end{array}$ & 8 & -36 & 2 & & 5.2 & \\
\hline $\begin{array}{l}\text { Left parahippocampal gyrus } \\
\qquad \text { (BA 27/30) }\end{array}$ & -8 & -34 & 0 & & 4.9 & \\
\hline Inferior occipital gyrus (BA I7/I8) & -26 & -96 & -8 & 1525 & 5.1 & 0.0001 \\
\hline Left posterior lateral cerebellum & -36 & -74 & -18 & & 4.7 & \\
\hline
\end{tabular}

BA, Brodmann area.

I. Uncorrected for multiple comparisons.

Table 2 Regions demonstrating functional connectivity with the left inferior frontal gyrus in patients

\begin{tabular}{llllll}
\hline Region & $X$ & $Y$ & $Z$ & $Z$ score & $\begin{array}{c}P \text { corrected } \\
\text { (voxel) }\end{array}$
\end{tabular}

\section{Positive correlations}

Left inferior frontal gyrus (BA 45/46)

Right superior frontal gyrus (BA 9)

Left superior temporal gyrus (BA 22)

Right middle temporal gyrus (BA 2I)

Right posterior lateral cerebellum

-54
36
-60
54
32

36

$-6$

7.I

0.0001

$\begin{array}{lll}56 & 28 & 4.7\end{array}$

0.001

0.01

0.005

0.01

Negative correlations

Right lingual gyrus (BA I7)

Posterior cingulate gyrus (BA 29)

10

$\begin{array}{rr}6 & 0 \\ -14 & -12\end{array}$

4.4

4.2

BA, Brodmann area.

All $P<0.05$, corrected for multiple comparisons (for spatial extent thresholded at $Z=3.2$ ).

and fusiform gyri, and in the precentral gyrus and the medial parietal lobe. This group did not show greater correlations in any area (Table 4$)$.

\section{DISCUSSION}

The main aim of this study was to examine the relationship between activity levels in of people with schizophrenia who were not prone to auditory hallucinations; this could have clarified whether the differential activation we observed was related specifically to the propensity to hallucinate, as opposed to the disorder of schizophrenia itself. There was no between-group difference in ratings of task performance outside the scanner. Ideally, functional imaging studies of cognitive tasks should involve the measurement of behavioural performance 'online', to assess how well participants are performing during image acquisition. However, covert speech cannot easily be measured without introducing additional and potentially undesirable cognitive demands. We sought to minimise the influence of variation in performance by training the participants on the tasks prior to scanning, obtaining ratings of their performance immediately after each task, and excluding those who reported or demonstrated marked difficulties with task execution. Moreover, both groups showed left inferior frontal cortical activation in association with the faster rate of generation of inner speech consistent with that seen in previous studies of inner speech (Frith et al, 1995; McGuire et al, 1996; Shergill et al, 2000b, 2001). This pattern of activation suggests that the participants were actually carrying out the tasks during the scanning.

\section{Generation of inner speech}

As predicted, increasing the rate of covert articulation was associated with activation in the left inferior frontal cortex. This was evident in all the participants, suggesting that the silent generation of inner speech is not impaired in people with a history of hallucinations; this is supported by findings from previous neuroimaging and cognitive psychology studies (Frith et al, 1995; McGuire et al, 1996; Shergill et al, $2000 b$ ). The consistent presence of significant left inferior frontal activation allowed us to generate a functional connectivity map for this region in each individual.

\section{Verbal self-monitoring}

The anticipated activation of the lateral temporal cortex in association with the faster rate of generation was evident in the healthy participants. Those in the patient group showed a significant attenuation of activation in the right (but not the left) superior temporal gyrus, the right parahippocampal gyrus and the right cerebellar cortex. These data are consistent 
Table 3 Patient $v$. control groups: differences during faster rate (categorical analysis)

\begin{tabular}{llllll}
\hline Region & $X$ & $Y$ & $Z$ & $\begin{array}{c}\text { Cluster size } \\
\text { (voxels) }\end{array}$ \\
\hline
\end{tabular}

Greater activation in patients during faster rate

Left lenticular nucleus $-2$

Greater activation in controls during faster rate

Right posterolateral cerebellum

Right parahippocampal gyrus (BA 27)

Right postcentral gyrus (BA I/2/3)

Right inferior parietal lobule (BA 40)

Right superior temporal gyrus (BA 42)

$\begin{array}{rrr}8 & -62 & -8 \\ 16 & -30 & -2 \\ 58 & -18 & 34 \\ 58 & -24 & 24 \\ 50 & -32 & 16\end{array}$

$\begin{array}{ll}36 I & 2.9 \\ I 57 & 3.8 \\ 822 & 4.0 \\ & 3.4 \\ & 3.3\end{array}$

BA, Brodmann area.

I. Uncorrected for multiple comparisons.

with a modulatory effect of left inferior frontal activity on activity in the temporal and cerebellar cortex in healthy individuals, and an attenuation of these modulatory effects in people with schizophrenia who are prone to auditory hallucinations. The interpretation is more complex in the case of the parahippocampal activation, which might have reflected 'deactivation' (i.e. greater activation at the slower rate) during the faster rate of covert articulation in the patients. However, in a previous fMRI study of auditory verbal imagery, we found similar differences between hallucination-prone patients and controls in these same three regions (Shergill et al, $2000 b$ ). In that study, these differences could have been related to the demands on semantic and phonological processing associated with imagining alien speech, but as the present study simply involved covert articulation of a single word, this explanation is unlikely.

The involvement of the right as opposed to the left temporal cortex is of interest, as the right temporal cortex has also been a site of differential activation in studies of verbal fluency in schizophrenia
(Spence et al, 2000), and this region appears to be more active than its left homologue when people are experiencing auditory hallucinations (Shergill et al, 2000). Although previous functional imaging studies have demonstrated involvement of the right temporal cortex in processing prosody (George et al, 1996) and inference of what is being said, as well as an emotional response to its content (Canli et al, 1998), this study suggests a more fundamental role in monitoring language production than previously thought and this may be reflected in the bilateral activation of the superior temporal gyri during the faster rate of covert articulation in healthy individuals.

A common feature of lateral temporal, parahippocampal and cerebellar cortex is that these regions are all implicated in cognitive self-monitoring (Frith \& Done, 1988; Gray et al, 1991; Blakemore et al, 1998). Lesion and neuroimaging studies suggest that the cerebellum acts as a 'comparator' in both motor and verbal tasks, comparing intended with actual performance and modulating cerebral cortical activity appropriately (Blakemore et al, 1998). The hippocampus has also been proposed as the 'comparator' in experimental models of cognitive self-monitoring (Gray et al, 1991), whereas the lateral temporal cortex has been more specifically implicated in the monitoring of inner speech (McGuire et al, 1996; Shergill et al, 2000b).

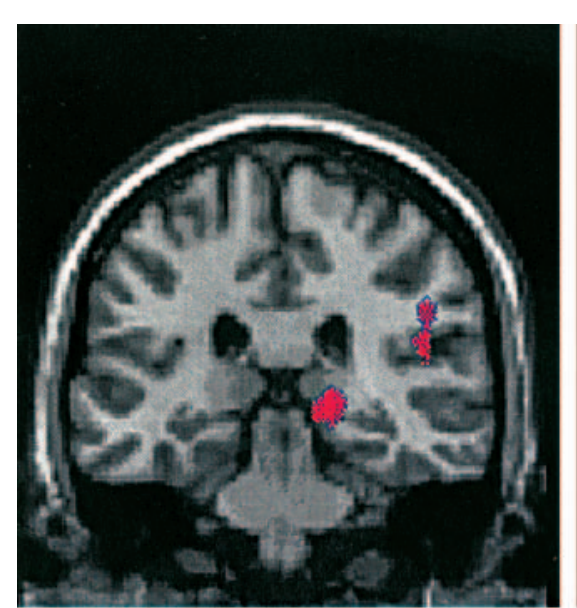

(a)

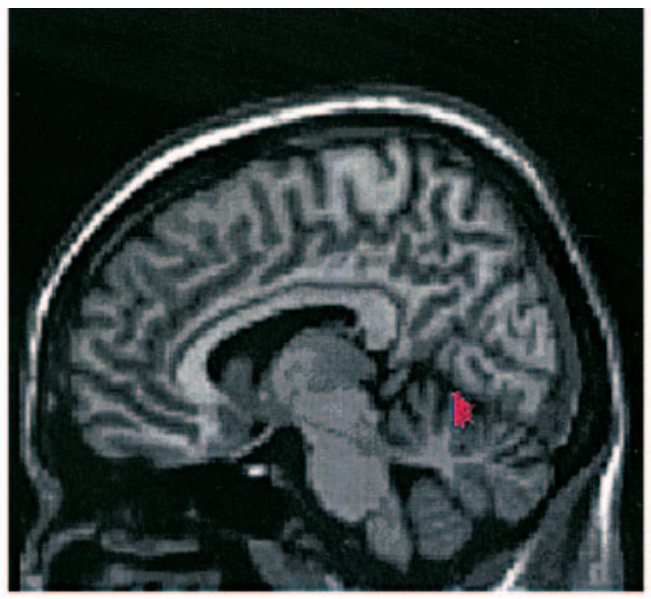

(b)

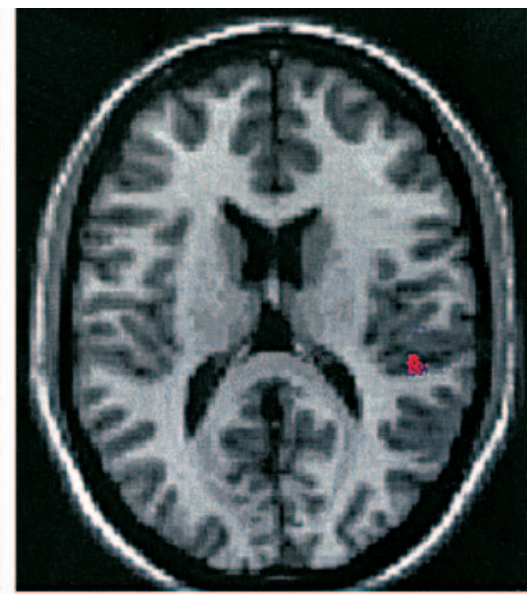

(c)

Fig. I Regions showing between-group differences with increased rate of generation of inner speech - subtraction analysis. Cortical regions showing greater activation during the faster rate of generation of inner speech (compared with slower rate) in the control group compared with the patient group are shown in red. Activation maps were rendered onto an individual $T_{1}$-weighted magnetic resonance imaging template and coordinates correspond to Talairach space $(X, Y, Z$ coordinates in mm). The three slices represent sections of the brain: (a) is a coronal section at $-30 \mathrm{~mm}$ illustrating differences in activation in the right parahippocampal gyrus and inferior parietal lobule/superior temporal gyrus; (b) is a sagittal section at $+8 \mathrm{~mm}$ showing activation in the right lateral cerebellar cortex; (c) is an axial section at $+16 \mathrm{~mm}$ showing activation in the right superior temporal gyrus. 
Table 4 Patient $v$. control groups: differences in functional connectivity with the left inferior frontal gyrus

\begin{tabular}{|c|c|c|c|c|c|c|}
\hline Region & $x$ & $Y$ & $z$ & $\begin{array}{l}\text { Cluster size } \\
\text { (voxels) }\end{array}$ & $Z$ score & $P$ (cluster) \\
\hline \multicolumn{7}{|l|}{ Correlations greater in controls } \\
\hline $\begin{array}{l}\text { Right parahippocampal/fusiform gyri } \\
\text { (BA 36/37) }\end{array}$ & 40 & -30 & -16 & 1689 & 3.6 & 0.0001 \\
\hline Right insula & 44 & -14 & 10 & 340 & 3.4 & $0.0 \mathrm{I}^{\mathrm{I}}$ \\
\hline Right precentral gyrus (BA 4/6) & 66 & -8 & 32 & 699 & 3.4 & 0.001 \\
\hline Left medial parietal lobe (BA 7) & -16 & -78 & 56 & 716 & 3.3 & 0.001 \\
\hline $\begin{array}{l}\text { Right superior temporal gyrus } \\
\text { (BA 22/38) }\end{array}$ & 44 & 0 & -10 & 194 & 3.2 & 0.05 \\
\hline $\begin{array}{l}\text { Right superior/middle temporal gyrus } \\
\text { (BA 22/2I) }\end{array}$ & 52 & 6 & -16 & & 3.1 & \\
\hline
\end{tabular}

BA, Brodmann area.

I. Uncorrected for multiple comparisons.

\section{Functional connectivity of the left inferior frontal gyrus}

The functional connectivity analyses generated maps of regions that showed BOLD activity that was significantly correlated with that in the maximal focus of activation within the left inferior frontal cortex, over the course of the experiment. This was an exploratory analysis, which sought to provide a more stringent temporal test of our hypotheses than the comparison of activity at fast and slow rates of covert articulation. In those with schizophrenia, activity in the left inferior frontal gyrus was positively correlated with activity in the left superior temporal gyrus, the right middle temporal gyrus and the right lateral cerebellum, providing further support for the involvement of these structures in a self-monitoring network. A positive modulation of temporal activity during verbal generation accords with data from a PET study of whispering at different rates (Paus et al, 1996), and from electrophysiological studies (Müller-Preuss \& Ploog, 1981; Creutzfeldt et al, 1989). However, when compared with the control group, left inferior frontal activity was less strongly correlated with activity in the right superior and middle temporal gyri, and the junction of the right parahippocampal/ fusiform gyri. The attenuation of correlation with activation in the right temporal cortex is consistent with the results of the categorical comparison discussed above, while the difference in the correlations with activity in the parahippocam$\mathrm{pal} /$ fusiform area was close (but lateral) to the parahippocampal site of differences in activation.
Functional connectivity between cortical grey-matter regions is mediated by axonal projections within cerebral white matter, and diffusion tensor MRI (Basser et al, 1994) - by measuring the diffusion characteristics of water molecules in tissue - permits evaluation of white-matter integrity and hence a more direct examination of brain connectivity. Some studies (Lim et al, 1999; Agartz et al, 2001), but not all (Steel et al, 2001), report reduced white-matter integrity in schizophrenia. However, as measurements were only possible in large white-matter regions or easily identified structures such as the corpus callosum, few studies have examined the frontal projections implicated in schizophrenia. However, a study examining the uncinate fasciculus (a major frontotemporal tract) found no direct difference between people with schizophrenia and a control group (Lawrie et al, 2002); however, the former failed to demonstrate the normal left-right (left greater than right) asymmetry evident in the healthy brains. This lends support to the lateralisation hypothesis proposed by Crow et al (1989) in which abnormal neural development of brain lateralisation is critical to the aetiology of schizophrenia. The attenuated activation within right-sided temporal and parietal structures evidenced by the people with schizophrenia in our study could be incorporated into this theory, which posits a disconnection between right-sided spatial or paradigmatic aspects of language and the linear (phonological) output restricted to the left hemisphere. The right-sided attenuation could be evidence of this disconnection, in the presence of normal left-sided language (function, which is suggested to be involved in the development of the nuclear symptoms of schizophrenia; the basis of which may lie in the difficulties that patients have with 'self' $v$. 'other' distinctions (Crow et al, 1989).

In summary, neuroimaging studies indicate that healthy people show activation in brain regions involved in speech generation (left inferior frontal cortex) and perception (temporal cortex) during the generation and monitoring of inner speech (Shergill et $a l$, 2001, 2002). Verbal self-monitoring seems to be particularly associated with activation in the temporal cortex bilaterally, the right parahippocampal cortex and the right lateral cerebellum (Shergill et al, 2001). The perception of auditory hallucinations in people with schizophrenia is associated with activation in the same parts of frontal and temporal cortices that are normally engaged during the generation and perception of inner speech, in the absence of any right cerebellar or parahippocampal activation (Shergill et al, 2000a). Moreover, when generating inner speech, people whose schizophrenia is in remission but who are prone to hearing 'voices' show relatively reduced activation in the temporal, right parahippocampal and right cerebellar cortex - areas implicated in verbal self-monitoring (Shergill et $a l, 2000 b$ ). Our study suggests that this is present even with generation of simple verbal stimuli. Thus, self-monitoring appears dysfunctional even in people whose disease is in remission. However, it is still unclear whether this putative failure of self-monitoring in schizophrenia is specific to the verbal modality or is also apparent during the planning or execution of other sensorimotor acts.

\section{ACKNOWLEDGEMENTS}

S.S.S. is supported by a Wellcome Advanced Fellowship. We thank Chris Andrew, Sophia Frangou and Andrew Simmons for technical support.

\section{REFERENCES}

Agartz, I., Andersson, J. L. R. \& Skare, S. (200I) Abnormal white matter in schizophrenia: a diffusion tensor imaging study. Neuroreport, 12, 225I-2254.

American Psychiatric Association (1994) Diagnostic and Statistical Manual of Mental Disorders (4th edn) (DSM-IV). Washington, DC: APA.

Annett, M. A. (1970) A classification of hand preference by association analysis. British Journal of Psychology, 61, 303-32I. 
Basser, P. J., Mattiello, J. \& Le Bihan, D. (1994) MR diffusion tensor spectroscopy and imaging. Biophysical Journal, 66, 259-267.

Blakemore, S. J., Wolpert, D. M. \& Frith, C. D. (1998) Central cancellation of self-produced tickle sensation. Nature Neuroscience, I, 635-640.

Canli, T., Desmond, J. E., Zhao, Z., et al (1998) Hemispheric asymmetry for emotional stimuli detected with fMRI. Neuroreport, 9, 3233-3239.

Creutzfeldt, O., Ojemann, G. \& Lettich, E. (1989)

Neuronal activity in the human lateral temporal lobe. II. Responses to the subject's own voice. Experimental Brain Research, 77, 475-489.

Crow, T. J., Ball, J., Bloom, S. R., et al (1989)

Schizophrenia as an anomaly of development of cerebral asymmetry. A postmortem study and a proposal concerning the genetic basis of the disease. Archives of General Psychiatry, 46, II45-II50.

Curio, G., Neuloh, G., Numminen, J., et al (2000) Speaking modifies voice-evoked activity in the human auditory cortex. Human Brain Mapping, 9, 183-191.

Deeke, L., Scheid, P. \& Kornhuber, H. H. (1969) Distribution of readiness potential, premotion positivity, and motor potential of the human cerebral cortex preceding voluntary finger movements. Experimental Brain Research, 7, 158-168.

\section{Erdler, M., Beisteiner, R., Mayer, D., et al (2000)} Supplementary motor area activation preceding voluntary movement is detectable with a whole-scalp magnetoencephalography system. Neuroimage, II, 697-707.

Frith, C. D. \& Done, D. J. (1988) Towards a neuropsychology of schizophrenia. British Journal of Psychiatry, 153, 437-443.

_ , Friston, K. J., Herold, S., et al (1995) Regiona brain activity in chronic schizophrenic patients during the performance of a verbal fluency task. British Journal of Psychiatry, 167, 343-349.

George, M. S., Parekh, P. I., Rosinsky, N., et al (1996) Understanding emotional prosody activates right hemisphere regions. Archives of Neurology, 53, 665-670.

Gray, J. A., Feldon, J., Rawlins, J. N. P., et al (1991)

The neuropsychology of schizophrenia. Behavioural and Brain Sciences, 14, I-19.

Lawrie, S. M., Buechel, S., Whalley, H. C., et al (2002) Reduced frontotemporal functional connectivity in schizophrenia associated with auditory hallucinations. Biological Psychiatry, 5I, 1008-1011.

Lim, K. O., Hedehus, M., Moseley, M., et al (1999) Compromised white matter tract integrity in schizophrenia inferred from diffusion tensor imaging. Archives of General Psychiatry, 56, 367-374.

McGuire, P. K., Silbersweig, D. A., Wright, I., et a (1996) The neural correlates of inner speech and auditory verbal imagery in schizophrenia: relationship to auditory verbal hallucinations. British Journal of Psychiatry, 169, 148-159.

Müller-Preuss, P. \& Ploog, D. (198I) Inhibition of auditory cortical neurons during phonation. Brain Research, 215, 61-76.

Nelson, H. E. (1991) National Adult Reading Test. Windsor: NFER-Nelson

Ogawa, S., Lee, T. M., Kay, A. R., et al (1990) Brain magnetic resonance imaging with contrast dependent on

\section{CLINICAL IMPLICATIONS}

- Auditory hallucinations in schizophrenia may arise through impaired processing of inner speech.

- The impairment in processing inner speech is at the level of verbal generation rather than conscious evaluation or labelling of auditory material.

- This impaired self-monitoring may also be the core mechanism underlying other symptoms in the motor and somatic areas, such as passivity phenomena in schizophrenia.

\section{LIMITATIONS}

- Because the study did not include a group of participants with schizophrenia who were not prone to auditory hallucinations, it is not possible to separate the effect of schizophrenia in general from the specific propensity to experience auditory hallucinations.

- There was no overt behavioural measure of task performance during image acquisition.

- The study would have benefited from the inclusion of an unmedicated patient group and a greater sample size.

SUKHWINDER S. SHERGILL, MRCPSych, MICHAEL J. BRAMMER, PhD, RIMMEI FUKUDA, MD, STEVEN C. R. WILLIAMS, PhD, ROBIN M. MURRAY, FRCPsych, PHILIP K. McGUIRE, MRCPsych, Institute of Psychiatry, London, UK

Correspondence: Dr S. S. Shergill, Division of Psychological Medicine, Institute of Psychiatry, De Crespigny Park, Denmark Hill, London SE5 8AF, UK. Tel: 0207848 0350; fax: 0207848 0350; e-mail: s.shergill@iop.kcl.ac.uk

(First received I3 September 2002, final revision 28 November 2002, accepted 9 December 2002)

blood oxygenation. Proceedings of the National Academy of Sciences of the USA, 87, 9868-9872.

Paus, T., Perry, D. W., Zatorre, R. J., et al (1996) Modulation of cerebral blood flow in the human auditory cortex during speech: role of motor-to-sensory discharges. European Journal of Neurscience, 8, 22362246.

Shergill, S. S., Brammer, M. J., Williams, S., et al (2000a) Mapping auditory hallucinations in schizophrenia using functional magnetic resonance imaging. Archives of General Psychiatry, 57, 1033-1038.

—, Bullmore, E.T., Simmons, A., et al (2000b) The functional anatomy of auditory verbal imagery in patients with auditory hallucinations. American Journal of Psychiatry, 157, 1691-1693.

_, _ , Brammer, M. J., et al (200I) A functional MR study of auditory verbal imagery. Psychological Medicine, 3I, $241-253$.
—, Brammer, M. J., Fukuda, R., et al (2002) Modulation of activity in temporal cortex during generation of inner speech. Human Brain Mapping, 16 219-227.

Spence, S. A., Liddle, P. F., Stefan, M. D., et al (2000) Functional anatomy of verbal fluency in people with schizophrenia and those at genetic risk. Focal dysfunction and distributed disconnectivity reappraised. British Journal of Psychiatry, 176, 52-60.

Steel, R. M., et al (200I) Diffusion tensor imaging (DTI) and proton magnetic resonance spectroscopy ('H MRS) in schizophrenic subjects and normal controls. Psychiatry Research Neuroimaging, 106, 161-170.

Wolpert, D. M., Ghahramani, Z. \& Jordan, M. I. (1995) An internal model for sensorimotor integration. Science, 269, 1880-1882. 